\title{
Special issue on Financial Forensics and Fraud Investigation in the Era of Industry 4.0
}

\author{
Thomas K. Dasaklis ${ }^{1}$. Veni Arakelian²
}

Accepted: 25 October 2021 / Published online: 16 November 2021

(c) The Author(s), under exclusive licence to Springer Nature Switzerland AG 2021

The next-generation Internet and Industry 4.0 applications are set to change the rules of the game in finance. In particular, disruptive technologies and applications like artificial intelligence, cloud technology, distributed ledger technologies, cryptocurrencies, and IoT offer new opportunities. However, financial crime continues to represent a billion-dollar business nowadays. Fraudulent transactions/operations, money laundering, identity theft, misappropriation of funds, bribery and corruption, and Ponzi schemes are just a few among various types of financial crime.

Blockchain is also becoming important in third-generation security. And since quite some time, Blockchain has increasingly been looked upon in an IoT context. Blockchain is designed as a basis for applications that involve transactions and interactions. These can include smart contracts (smart contracts are automatically carried out when a specific condition is met, for instance, regarding the conditions of goods or environmental conditions) or other smart applications that support specific IoT processes. This way, blockchain technology can improve compliance in the IoT and IoT features and cost efficiency. The rapid development of Blockchain as a financial platform in different industries and the massive demand for its features and technological solutions created a broad view of the use of technology in business. As almost every industry uses some flexible practices for record keeping, it is not unreasonable to expect this technology to be implemented in a wide range of applications. With what started as a code posted by an anonymous developer to create a new currency platform, Blockchain has gained tremendous popularity, with almost every industry such as trade, shipping, health, public services, contributing to both saving resources as well as in creating new professions and jobs, contributing to economic growth and stability in global markets.

Thomas K. Dasaklis

dasaklis@eap.gr

Veni Arakelian

veniarakelian@yahoo.com

1 School of Social Sciences, Hellenic Open University, Patras, Greece

2 Economic Research and Investment Strategy, Piraeus Bank, Athens, Greece 
Given that financial services will be more affected by the new technology, it confirms that it is a financial innovation with multiple benefits for citizens and businesses. Its main feature is that transactions between the parties occur without a third party but using encryption techniques, ensuring security, transparency, and privacy. By the way, this achieves a fair distribution of resources without any intervention. On the other hand, the lack of a mainly regulatory framework for applying new technology is a risk, especially in market turmoil. Furthermore, cryptocurrencies have become a part of our lives. A lot of studies evaluate the diversification or the hedging benefits of cryptocurrencies when forming a portfolio allocation.

The objective of this special issue is an interdisciplinary discussion on the novel phenomenon of Blockchain and collaborative analysis of its economic consequences from game-theoretic, statistical, and financial points of view.

Publisher's Note Springer Nature remains neutral with regard to jurisdictional claims in published maps and institutional affiliations. 